\title{
Game Theoretic Analysis of Wireless Access Network Selection: Models, Inefficiency Bounds, and Algorithms
}

\author{
Matteo Cesana \\ DEI, Politecnico di Milano \\ piazza Leonardo da Vinci 32 \\ Milano, Italy \\ cesana@elet.polimi.it
}

\author{
Nicola Gatti \\ DEI, Politecnico di Milano \\ piazza Leonardo da Vinci 32 \\ Milano, Italy \\ ngatti@elet.polimi.it
}

\author{
Ilaria Malanchini \\ DEI, Politecnico di Milano \\ piazza Leonardo da Vinci 32 \\ Milano, Italy \\ malanchini@elet.polimi.it
}

\begin{abstract}
This paper addresses the problem of network selection, which arises when wireless mobile users can choose among multiple available wireless access networks to connect to. In particular, we are interested in studying the dynamics of the competition among different selfish mobile users which operate the network selection with the goal of minimizing their own selection cost. To this extent, we formalize the problem as a non-cooperative game, and we study the quality of the corresponding Nash equilibria under three expressions for the users' selection cost by deriving tight analytical bounds on the price-of-anarchy and the price-of-stability for the games. Finally, we introduce a mathematical programming formulation for the games which allows one to compute the equilibria in realistic wireless access network topologies.
\end{abstract}

\section{Categories and Subject Descriptors}

C.2.1 [Computer-Communication Networks]: Network Architecture and Design-Network communications

\section{General Terms}

Algorithms, Theory, Economics

\section{Keywords}

Congestion games, network selection, price-of-anarchy

\section{INTRODUCTION}

The world of wireless networking has experienced dramatic changes in the last decade due to the diffusion of multiple and more and more performing wireless technologies. In this scenario, mobile users have nowadays the opportunity of choosing among multiple wireless access networks. Indeed, $2 \mathrm{G}$ cellular systems have recently been complemented by $3 \mathrm{G}$ cellular systems, which, in many situations, are deployed side by side with multi-domain Wireless Local Area

Permission to make digital or hard copies of all or part of this work for personal or classroom use is granted without fee provided that copies are not made or distributed for profit or commercial advantage and that copies bear this notice and the full citation on the first page. To copy otherwise, to republish, to post on servers or to redistribute to lists, requires prior specific permission and/or a fee.

GameComm 2008 October 20, 2008, Athens, GREECE.

Copyright 2008 ICST 978-963-9799-31-8.
Networks (WLANs) as well as Wireless Mesh Networking (WMN) infrastructures.

This plethora of coexisting/cooperating wireless access systems, which often in the literature goes under the name of $4 G$ Networks, may be potentially exploited by mobile users to achieve seamless, ubiquitous, and pervasive connectivity. On the down side, in order to reach this ambitious goal, heterogeneous challenges still have to be addressed in different fields.

From the network point of view, new solutions are required to effectively handle the user's handover among multiple networks, ${ }^{1}$ and to manage the radio resource allocation process. On the mobile user's side, the main challenge deals with designing proper solutions to drive the network selection, that is, the dynamic and automatic choice of the "best" wireless access network to connect to. This calls for enhanced solutions to monitor and classify the multiple connectivity opportunities (e.g., based on the achievable throughput, the wireless interference level, etc.), and to automatically take decisions to actually select the "best" networks on the basis of the aforementioned measures [11].

In this work, we resort to non-cooperative game theory to model the problem of network selection, where mobile users selfishly compete to minimize their own cost. Namely, we introduce a non-cooperative game theoretic framework which belongs to the class of congestion games. Since in these settings the exact cost of the users depends on the actual throughput and its definition is very involved and cannot be employed in a game model, we use approximations based on interference and nominal throughput. More precisely, we study the quality of the corresponding Nash equilibria under three expressions for the user's cost; as a first step we define a purely interference-based cost function, which leads to a non-cooperative game where users choose the least interfered access network; then we address those cases where cost also depends on the nominal achievable throughput. In this last scenario, we study two multi-objective utility functions endorsing both interference and nominal throughput.

In all the aforementioned network selection games, we derive non-trivial upper bounds for the price-of-anarchy (PoA, see [12]) and the price-of-stability (PoS, see [2]) which are significantly tighter than those available in the literature. Finally, we propose a mathematical programming model to numerically characterize the quality of the Nash equilibria, thus providing an operational tool to "solve" the game. The work will be completed in future by simulating (e.g., by NS2

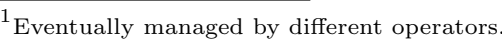


simulator) the actual throughput achievable employing each specific cost function.

The paper is organized as follows: in Section 2, we set the background for our work by reviewing previously published approaches in the field of network selection, highlighting the novelty of the proposed contribution. Section 3 defines the game theoretic problem. In Section 4, we report the main game theoretic results considering three different cost functions. In Section 5 we provide a mathematical programming formulation to compute equilibria and then we experimentally compare them. Concluding remarks and comments on ongoing related activities are reported in Section 6. Appendices $\mathrm{A}$ and $\mathrm{B}$ report some applications of the algorithms proposed in the paper.

\section{BACKGROUND AND CONTRIBUTIONS}

In the field of network selection, the research efforts have mainly focused on two major aspects: first, the definition of novel metrics to measure the perceived quality of accessing users to steer the selection decisions, second, the design of communication protocols customized to the multinetwork scenario. References [8] and [15] fall in the former research track, and propose realistic measures of the user's QoS, which can be consequently used to drive the selection phase. Recently, Song and Jamalipour [17] address the problem of network selection resorting to mathematical modelling and computing techniques. Namely, the authors propose to use Grey Relational Analysis and Analytic Hierarchy Processing to determine the utility related to different selection choices.

Research of the latter track usually focuses on specific network scenarios (technologies). Lee and Miller address in [13] the problem of selecting among several 802.11-based access points, by proposing an effective solution to distribute roaming information to the end users, which can be used to discriminate in the selection phase. The same scenario with multiple 802.11 access points is considered in [6], where the authors study the load balancing among the different access points by steering the end user decisions while accounting both for user preferences and network context. Bernaschi et al. [5] propose a vertical handover protocol to handle the user mobility between WLAN and cellular systems.

In our previous work [7], we focus on the joint problem of resource allocation and network selection which is modelled as a two-stage game. On the other side, in this paper we are interested in gathering general results which capture the dynamics of competition which is imbedded in the process of network selection. We resort to non-cooperative game theoretic models, which constitute a powerful modeling/analysis framework to represent situations where decision makers (mobile users, in our case) take specific actions that may have mutual and conflicting influence. To characterize stable outcome of the game, we leverage the concept of Nash equilibrium, which could be qualitatively defined as a status of the game where each player has no incentive in unilaterally deviating from the played strategy [10].

However, provided that a game always admits Nash equilibria, it is fundamental to find such equilibria and characterize them. Indeed, a game may possess several equilibria of different "quality". To this extent, and to characterize such quality of Nash equilibria, we use the well known concepts of price-of-anarchy (PoA) and price-of-stability (PoS), defined as the ratio between the "value" of the best/worst equilibrium, respectively, and the optimal solution. Commonly, the value considered in literature is the social cost, i.e. the sum of the costs of all the users.

Starting from the aforementioned background, the main contributions of the present paper are the following.

Models. The formalization of the network selection problem with a non-cooperative game theoretic framework under different user's cost functions. Namely, we consider three cost functions for the end users, and cast the corresponding game as a congestion game.

Inefficiency bounds. The derivation of non-trivial upper bounds for the PoA and the PoS of the proposed games which are significantly tighter than the bounds known in the literature. Furthermore, the bounds we provide are applicable to a large class of congestion games.

Algorithms. The introduction of Nash equilibria characterization techniques based on non-deviation inequalities and mathematical programming to numerically derive the equilibria for the proposed games.

\section{PROBLEM STATEMENT}

We study the scenario composed of a WiFi network with $m$ access points and $n$ users, where each user can choose the access point to connect to. We denote by $\mathcal{A}$ the set of access points and by $\mathcal{N}$ the set of users. We model this scenario as a non-cooperative game in which users are players and their available actions are the selection of an access point among the available ones. We denote by $A_{i} \in \mathcal{A}$ the $i$-th access point. Each access point $A_{i}$ is characterized by a frequency $f_{i}$ on which it transmits and by a coverage area, i.e. the area covered by the transmission range of the access point.

The topology of the network specifies the number $m$ of access points, their positions, their frequencies, and their coverage areas. In this paper we assume both that the frequencies $f_{i}$ s are a given data of the problem (therefore they will be considered as parameters of the game) and that there is not any pair of access points with the same frequency such that their coverage areas overlap. These assumptions allow one to have a tractable expression for the users' interference, which, formally, can be defined as the number of users connected to the chosen access point $A_{i}$.

The actions available to a user are defined by the topology of the network and by the user's position. Precisely, each user can select one access point among all the ones whose coverage areas cover the user's position. In our game theoretical analysis we consider the coverage areas as a given data, while in the experimental setting discussed in Section 5 we will adopt a specific propagation model. For the sake of clarity, given the network topology, we differentiate users on the basis of the access points they can connect to. We denote by $N_{l} \in \mathcal{N}$ where $l \subseteq\{1, \ldots, m\}$ the set of users that can connect to any access point whose subscript occurs in $l$, e.g. $N_{1,2}$ is the set of users that can connect to $A_{1}$ and $A_{2}$. We denote by $n_{l}$ the cardinality of $N_{l}$. We report an example of network in Fig. 1, where $m=2, n_{1}=4, n_{2}=3$, and $n_{1,2}=3$. Black circles denote users and lines between users and access points denote connections.

Each user $j$ has a cost function $c_{j}\left(i, x^{i}\right)$ that depends on access point $A_{i}$ it connects to and on congestion $x^{i}$ of access point $A_{i}$. Customarily, we assume $c_{j}\left(i, x^{i}\right)$ to be strictly monotonically increasing in $x^{i}$. We assume each user to be 


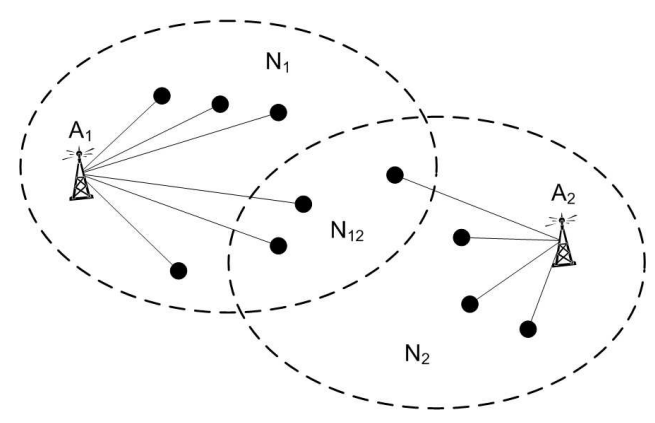

Figure 1: A network with two access points.

rational and to behave selfishly trying to minimize her cost. Finally, as common in game theoretic studies for communication network, we assume information to be complete.

We denote by $x_{l}^{i}$ the number of users belonging to $N_{l}$ that connect to access point $A_{i}$. A solution to the problem we are dealing with is a Nash equilibrium, i.e. strategy profile $S^{*}=\left(s_{1}, \ldots, s_{n}\right)$ such that no user can reduce her cost by deviating unilaterally from $S^{*}[10]$. In this paper we consider non-weighted congestion settings, i.e. each user congests resources with the same weight, formally, $x^{i}=\sum_{l} x_{l}^{i}$.

The game we are considering is a congestion game [16] that is: asymmetric (different users can have different available actions), single-choice (each user can select only one access point), and with player-specific cost functions (each user can have a different cost function). Rigorously speaking, this game is not a crowding game [14], i.e. a symmetric single-choice congestion game with player-specific cost functions that are monotonically increasing in the level of congestion, anyway it can be easily reduced to that. Indeed, we can build an auxiliary game, equivalent to the original, that is a crowding game. The auxiliary game is such that each agent can connect to all the access points and the cost for a user of connecting to the access points non-allowed in the original game is set equal to infinity. The equivalence between the original game and auxiliary one is straightforward. This equivalence leads to a prominent property: since all the users have the same weight in congesting the resources, the game is proved to always admit a Nash equilibrium in pure strategies [14]. Therefore, independently of the specific definition of $c_{j}$, we can focus on solving algorithms that limit their search to pure strategy equilibria.

In the next section, we consider three different definitions of cost functions and we analyze the corresponding games in terms of price-of-anarchy and price-of-stability. The objective function we use is the social cost. Subsequently, we will propose a mathematical programming formulation to compute Nash equilibria in real-world settings and we experimentally compare the equilibria with different cost functions in terms of interference and nominal throughput.

\section{GAME-THEORETIC ANALYSIS}

\subsection{Interference-based Cost Function}

We consider the case in which the cost functions depend only on the number of interferers. Formally, we have $c_{j}\left(i, x^{i}\right)=$ $x^{i}$. With these cost functions the game is essentially a singlechoice asymmetric congestion game, all users' cost functions being the same. This game admits an exact potential function that is the one provided by Rosenthal in [16], formally:

$$
\Psi(S)=\sum_{i=1}^{m} \sum_{k=1}^{x^{i}} k,
$$

where $S$ is the users' strategy profile. ${ }^{2}$

\subsubsection{Non-Deviation Inequalities Based Analysis}

In these simple settings, a Nash equilibrium can be found in closed-form by studying non-deviation inequalities and by solving them. This analysis is of paramount importance for the determination of the exact bounds on price-of-anarchy and price-of-stability and provides an effective tool for the characterization of the equilibria. We basis our analysis on Algorithm 1 and we apply it to a simple example.

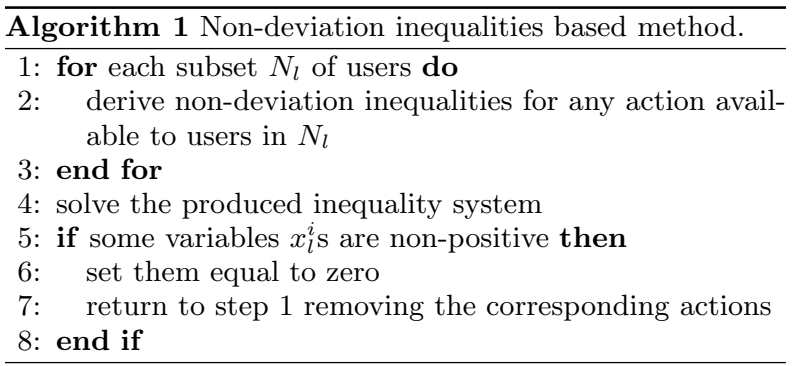

We apply Algorithm 1 to the topology depicted in Fig. 2. The number of users and their positions are considered as parameters. The variables are $x_{1,2}^{1}, x_{1,2}^{2}, x_{2,3}^{2}$, and $x_{2,3}^{3}$.

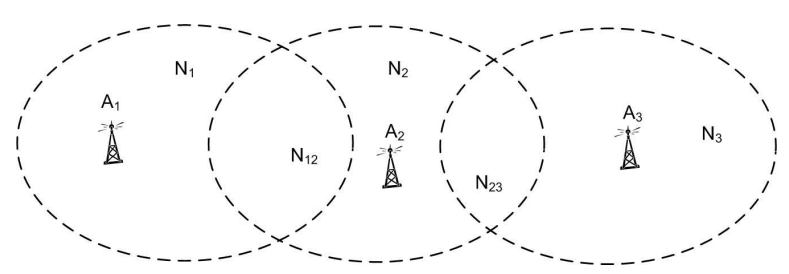

Figure 2: Reference scenario.

Initially, we write non-deviation inequalities under the assumption that no action was removed and then that at the equilibrium $x_{l}^{i}>0$ for all $i$ and $l$. This assumption will be verified once a solution to the produced inequality system was found. The non-deviation inequalities are:

$$
\left\{\begin{array}{l}
n_{1}+x_{1,2}^{1} \leq n_{2}+x_{1,2}^{2}+x_{2,3}^{2}+1 \\
n_{2}+x_{1,2}^{2}+x_{2,3}^{2} \leq n_{1}+x_{1,2}^{1}+1 \\
n_{2}+x_{1,2}^{2}+x_{2,3}^{2} \leq n_{3}+x_{2,3}^{3}+1 \\
n_{3}+x_{2,3}^{3} \leq n_{2}+x_{1,2}^{2}+x_{2,3}^{2}+1
\end{array} .\right.
$$

The solutions are the following (the calculations are provided in Appendix A).

- If $n$ is divisible by 3 , solutions can have two different social costs. The solution with the minimum social cost is: $x_{1,2}^{1}=\frac{n}{3}-n_{1}, x_{1,2}^{2}+x_{2,3}^{2}=\frac{n}{3}-n_{2}$, and $x_{2,3}^{3}=\frac{n}{3}-n_{3}$. Solutions with a larger social cost are of the form: $x_{1,2}^{1}=\frac{n-3}{3}-n_{1}, x_{1,2}^{2}+x_{2,3}^{2}=\frac{n}{3}-n_{2}$, and $x_{2,3}^{3}=\frac{n+3}{3}-n_{3}$.

\footnotetext{
${ }^{2}$ We recall that in a potential game every strategy profile that minimizes the potential function is a Nash equilibrium.
} 
- If $n+1$ is divisible by 3 , only solutions with the same social costs are possible. They are of the form: $x_{1,2}^{1}=$ $\frac{n+1}{3}-n_{1}, x_{1,2}^{2}+x_{2,3}^{2}=\frac{n+1}{3}-n_{2}$, and $x_{2,3}^{3}=\frac{n-2}{3}-n_{3}$.

- If $n-1$ is divisible by 3 , only solutions with the same social costs are possible. They are of the form: $x_{1,2}^{1}=$ $\frac{n-1}{3}-n_{1}, x_{1,2}^{2}+x_{2,3}^{2}=\frac{n-1}{3}-n_{2}$, and $x_{2,3}^{3}=\frac{n+2}{3}-n_{3}$.

In order to verify the assumption $x_{l}^{i}>0$ for all $i$ and $l$, we need to compute $x_{1,2}^{2}=n_{1,2}-x_{1,2}^{1}$ and $x_{2,3}^{3}=n_{2,3}-x_{2,3}^{2}$. If the assumption is verified, then we have found one or more equilibria. Otherwise, we set the variables that violate the above assumption to be equal to zero and we produce an inequality system removing the actions corresponding to the variables set equal to zero. For instance, if $x_{2,3}^{3}<0$, we set it equal to zero and we remove the possibility of choosing $A_{3}$ for all users in $N_{2,3}$. That is, all the users in $N_{2,3}$ will choose $A_{2}$, formally, $x_{2,3}^{2}=n_{2,3}$. In this case the new problem to solve is simpler since both the number of variables and the number of inequalities decrease by two. We call $n^{\prime}=n_{1}+n_{1,2}+n_{2}+n_{2,3}$. The non-deviation inequality system is:

$$
\left\{\begin{array}{l}
n_{1}+x_{1,2}^{1} \leq n_{2}+x_{1,2}^{2}+n_{2,3}+1 \\
n_{2}+x_{1,2}^{2}+n_{2,3} \leq n_{1}+x_{1,2}^{1}+1
\end{array} .\right.
$$

The solutions are the following (the calculations are provided in Appendix A).

- If $n^{\prime}$ is divisible by 2 , only one solution is possible. It is: $x_{1,2}^{1}=\frac{n^{\prime}}{2}-n_{1}$ and $x_{1,2}^{2}=\frac{n^{\prime}}{2}-n_{2}-n_{2,3}$.

- If $n^{\prime}+1$ is divisible by 2 , only solutions with the same social cost are possible. They are of the form: $x_{1,2}^{1}=$ $\frac{n^{\prime}+1}{2}-n_{1}$ and $x_{1,2}^{2}=\frac{n^{\prime}-1}{2}-n_{2}-n_{2,3}$.

If $x_{1,2}^{1}$ and $x_{1,2}^{2}$ are strictly greater than zero, we have found one or more equilibria. Otherwise, we must set the variable that is negative equal to zero and we must produce a new inequality system. The application of the algorithm continues as done above.

\subsubsection{Bounds on PoA and PoS}

We analyze now price-of-anarchy (PoA) and price-of-stability (PoS) with objective function $F$ defined as the social cost, formally, $F=\sum_{i=1}^{m}\left(x^{i}\right)^{2}$. We analyze both the settings when $n$ is finite (atomic congestion game model) and when $n$ is infinite (non-atomic congestion game model [18]) and therefore the contribution of each user to the congestion is negligible. We analyze this second setting to provide the asymptotic properties of the considered model as the number of users goes to infinity. We state the following proposition.

Proposition 4.1. The exact upper bounds for PoA and PoS are: $\operatorname{PoA}(n<\infty) \leq 1 . \overline{6}, \operatorname{PoA}(n=\infty)=1, \operatorname{PoS}(n<$ $\infty)=1$, and $\operatorname{PoS}(n=\infty)=1$.

Proof. We start by proving the bounds on PoS. The proof is based on the potential function. When $n<\infty$, we have that the strategy profile that minimizes $\Psi$ minimizes also $F$. Formally, $\arg \min \Psi=\arg \min F$, indeed $\arg \min \Psi=$ $\arg \min \sum_{i=1}^{m} \sum_{k=1}^{x^{i}} k=\arg \min \sum_{i=1}^{m} \frac{x^{i} \cdot\left(x^{i}+1\right)}{2}=\arg \min$ $\sum_{i=1}^{m}\left(x^{i}\right)^{2}$. Therefore, when $n<\infty$ we have $\operatorname{PoS}=1$. When $n=\infty$, the game admits potential function

$$
\Psi(S)=\sum_{i=1}^{m} \int_{0}^{x^{i}} k d k=\sum_{i=1}^{m} \frac{1}{2}\left(x^{i}\right)^{2}
$$

that is equal (apart a multiplicative factor $\frac{1}{2}$ ) to $F$ and then they are minimized by the same argument. It follows that also when $n=\infty$ we have $\operatorname{PoS}=1$.

We now prove the bounds on PoA. We recall that when $n=\infty$ any congestion game admits equilibria that are all equivalent for what concerns the social cost [4]. Therefore, the worst equilibrium when $n=\infty$ gives a social cost that is exactly the one given by the best equilibrium. It easily follows that, since $\operatorname{PoS}=1$, also $\operatorname{PoA}=1$. When $n<\infty$, the proof is not straightforward. We recall that the most severe bound on PoA presented in literature for a generic congestion game with linear cost functions is 2.5 [3]. We show that this bound can be significantly reduced to $1 . \overline{6}$ and that this last bound is exact. Indeed, we provide a case in which PoA is exactly $1 . \overline{6}$.

The proof is organized in two parts. In the first part of the proof, we show that the worst topology class is the "corridor", i.e. the topology in which the coverage area of each access point overlaps the coverage areas of at most two access points. An example is reported in Fig. 3. In the second part of the proof, we show that among all the possible "corridor" topologies, the worst one for the PoA is when $m=3$ and $n=3$.

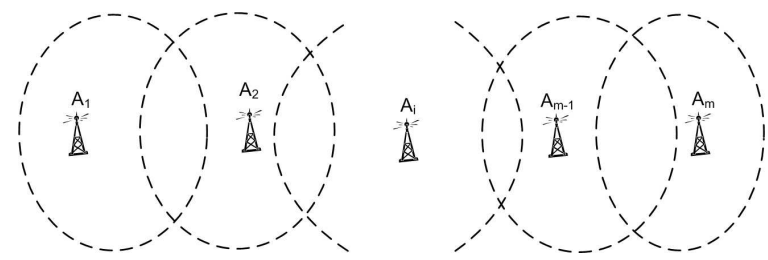

Figure 3: The "corridor": the worst topology class for PoA.

We consider the first part of the proof. By definition, PoA is equal to

$$
\operatorname{PoA}=\begin{array}{cc}
\max & \sum_{i}\left(x^{i}\right)^{2} \\
\text { s.t. } & f_{j}\left(x_{l}^{1}, \ldots, x_{l}^{m}\right)=0 \\
e_{j}\left(x_{l}^{1}, \ldots, x_{l}^{m}\right)=0
\end{array},
$$

where $f_{j}\left(x_{l}^{1}, \ldots, x_{l}^{m}\right)=0$ are the feasibility constraints of the form $\sum_{l} x_{l}^{i}=n_{l}$ (i.e., all the users have been assigned to exactly one access point) and $e_{j}\left(x_{l}^{1}, \ldots, x_{l}^{m}\right)=0$ are the equilibrium constraints of the form $x_{l}^{i} \leq x_{l}^{j}+1$ with $i \neq j$. We notice that numerator and denominator in PoA differ, apart for max and min, for the presence in the numerator of the constraints $e_{j}$ s. Obviously, fixed $m$ and $n$, the lower the number of constraints $e_{j}$ s the greater the value of the numerator. As a result, fixed $m$ and $n$, the PoA is maximum when the number of constraints $e_{j} \mathrm{~s}$ is minimum. The number of constraints $e_{j}$ s depends on the number of overlaps between access points' coverage areas. Precisely, a couple of constraints $e_{j}$ s is introduced for each pair of overlapping coverage areas. It can be easily shown that the topology class that minimizes the number of constraints is the "corridor".

We consider the second part of the proof. We exclude the cases in which $m=1$ and $m=2$, since it can be shown, by employing Algorithm 1, that in these cases PoA is equal to 1 . The worst situation for PoA is when $n$ is multiple of $m$ (formally, $n=\alpha \cdot m$ with $\alpha \in \mathbb{N}$ ) and the assignment $x^{i}=n / m$ for all $i$ is feasible. Essentially, if $n$ is a multiple of 
$m$ and the assignment $x^{i}=n / m$ is not feasible, PoA can be increased by rearranging the positions of the users to make feasible the assignment $x^{i}=n / m$ for all $i$. If instead $n$ is not a multiple of $m$, PoA can be increased by adding or removing $n^{\prime}$ users to make $n+n^{\prime}$ or $n-n^{\prime}$ to be a multiple of $m$. When $n$ is a multiple of $m$ and the assignment $x^{i}=n / m$ is feasible, this assignment is the one that minimizes the social cost. Obviously, it is also the optimal Nash equilibrium. Equilibria different from the optimal solution are such that $x^{i} \in\{n / m-1, n / m, n / m+1\}$ for all $i$. A large part of these equilibria gives the same social costs. We are interested only in the equilibria that give different social costs. It can be shown that equilibria with different social costs have a different number of pairs $\left(x^{i}=n / m-1, x^{j}=n / m+1\right)$. The minimum number of these pairs is obviously 1 , whereas the maximum number is $\left\lfloor\frac{m+1}{4}\right\rfloor$. We can now calculate PoA. It is:

$$
\begin{aligned}
& \text { PoA }=\max _{\substack{i \in\{3, \ldots, m\} \\
m \cdot\left(\frac{n}{m}\right)^{2}}} \begin{array}{r}
\frac{\left\lfloor\frac{i+1}{4}\right\rfloor\left(\left(\frac{n}{m}-1\right)^{2}+\left(\frac{n}{m}+1\right)^{2}\right)+\left(m-2\left\lfloor\frac{i+1}{4}\right\rfloor\right) \cdot\left(\frac{n}{m}\right)^{2}}{}=\max _{i \in\{3, \ldots, m\}} 2\left\lfloor\frac{i+1}{4}\right\rfloor \frac{m}{n^{2}}+1 .
\end{array}
\end{aligned}
$$

Obviously, PoA is maximum when $i$ is maximum, i.e. $\mathrm{PoA}=2\left\lfloor\frac{m+1}{4}\right\rfloor \frac{m}{n^{2}}+1$. Moreover, PoA is maximum for the values of $m$ such that $m+1$ is a multiple of 4 . We obtain PoA $=2 \frac{m+1}{4} \frac{m}{n^{2}}+1$. Since $n=\alpha \cdot m$ with $\alpha \in \mathbb{N}$, we have $\mathrm{PoA}=2 \frac{m+1}{4} \frac{m}{\alpha^{2} \cdot m^{2}}+1=\frac{m+1}{2 m \cdot \alpha^{2}}+1$. Since $m \geq 3$, we have that the maximum value of PoA is when $m=3$ and $\alpha=1$, and therefore $n=3$. The worst $\mathrm{PoA}$ is $\frac{5}{3}=1 . \overline{6}$. Fig. 4 depicts a setting in which PoA is exactly $1 . \overline{6}$.

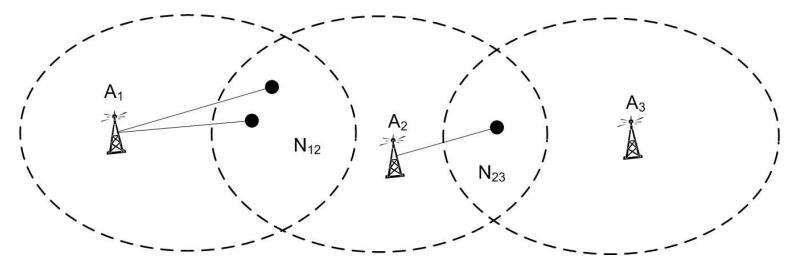

Figure 4: Worst case for PoA with interference based cost functions.

\subsection{Additive Interference-Rate Cost Function}

We consider the case in which the cost functions are linear combination of the number of interferers and the reverse of the rate perceived by users. We denote by $T_{j}^{i}$ the reverse of the rate perceived by user $j$ to connect to access point $A_{i}$. Users' cost functions are: $c_{j}\left(i, x^{i}\right)=\lambda_{1} x^{i}+\lambda_{2} T_{j}^{i}$ where $\lambda_{1}, \lambda_{2} \in \mathbb{R}_{+}$are user independent parameters. In other words, we are assuming that all the users use the same values of $\lambda_{1}$ and $\lambda_{2}$. Notice that, when $\lambda_{2}=0$, the game is exactly the one discussed in the previous section, whereas, when $\lambda_{1}=0$, there is not any competition among users. Indeed, in this second case, each user's cost function does not depend on the actions undertaken by the other users. In this situation, each user $j$ 's behavior is greedy trying to minimize $T_{j}^{i}$.
The game is properly a crowding game, since cost functions are player-specific. Furthermore, the game admits potential function:

$$
\Psi(S)=\lambda_{1} \sum_{i=1}^{m} \sum_{k=1}^{x^{i}} k+\lambda_{2} \sum_{j} T_{j}^{i} .
$$

We analyze PoA and PoS in this specific setting, with objective function $F$ defined as social cost. We state the following proposition.

Proposition 4.2. The exact upper bounds for PoA and PoS are: $\operatorname{PoA}(n<\infty) \leq 1 . \overline{6}, \operatorname{PoA}(n=\infty)=1, \operatorname{PoS}(n<$ $\infty)<1 . \overline{3}$, and $\operatorname{PoS}(n=\infty)=1$.

Proof. We start by proving PoS. When $n<\infty$, PoS being defined as

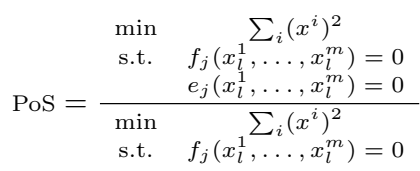

(the constraints $f_{j} \mathrm{~s}$ and $e_{j} \mathrm{~s}$ are defined in the previous section), the maximum value for PoS is when the constraints' number is maximum. (The reasoning is the reverse of the one we produced for PoA in the previous section.) It easily follows that the worst topology class is the one in which the overlap of all the coverage areas is not empty. We consider the setting depicted in Fig. 5 where $m$ and $n$ are parameters. There is one user in $N_{1}$ and $n-1$ users in $N_{1, \ldots, m}$.

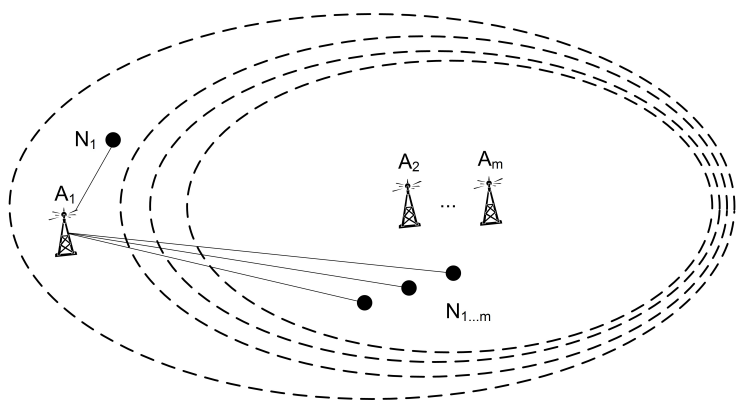

Figure 5: Worst case for PoA with additive interference-rate cost functions.

We assume that all the $n-1$ users in $N_{1, \ldots, m}$ are the same in terms of rate. We denote by $a$ the user in $N_{1}$ and by $b$ the users in $N_{1, \ldots, m}: T_{a}^{1}$ is the reverse of rate perceived by $a$ from the connection with $A_{1}, T_{b}^{i}$ with $i \in\{1, \ldots, m\}$ is the reverse of rate perceived by $b$ from the connection with $A_{i}$. We assume that $\lambda_{1} n+\lambda_{2} T_{b}^{1}<\lambda_{1}+\lambda_{2} T_{b}^{i}$ for all $i \in\{2, \ldots, m\}$. That is, there is a unique equilibrium where $x^{1}=n$ and $x^{i}=0$ for all $i \in\{2, \ldots, m\}$. It can be easily shown that this is the worst topology for PoS. In order to maximize PoS, we can assume all $T_{b}^{i}$ with $i \in\{2, \ldots, m\}$ to be the same and we denote them by $T_{b}^{-1}$. The optimal assignment that maximizes $\operatorname{PoS}$ is when $x^{i}=1$ for all $i$ (we omit the mathematics, it can be derived by calculating the derivative of PoS with respect to the number of users for each access point). We can write $\mathrm{PoS}$ as

$$
\mathrm{PoS}=\frac{\lambda_{1} n^{2}+\lambda_{2}\left(T_{a}^{1}+(n-1) T_{b}^{1}\right)}{\lambda_{1} n+\lambda_{2}\left(T_{a}^{1}+(n-1) T_{b}^{-1}\right)} .
$$


Since we have that $\frac{\lambda_{1}}{\lambda_{2}}<\frac{T_{b}^{-1}-T_{b}^{1}}{n-1}$, if $\lambda_{2}>0$, we can write:

$$
\operatorname{PoS}<\frac{\frac{T_{b}^{-1}-T_{b}^{1}}{n-1} n^{2}+T_{a}^{1}+(n-1) T_{b}^{1}}{\frac{T_{b}^{-1}-T_{b}^{1}}{n-1} n+T_{a}^{1}+(n-1) T_{b}^{-1}} .
$$

From the derivative of the bound with respect to $n$, i.e.

$$
\frac{d \mathrm{PoS}}{d n}=\frac{\left(T_{b}^{-1}-T_{b}^{1}\right) \cdot\left(-n^{2} T_{b}^{-1}+2 n T_{b}^{-1}-T_{b}^{1}\right)}{\left(-n T_{b}^{-1}-T_{b}^{1} n+T_{a}^{1} n-T_{a}^{1}+n^{2} T_{b}^{-1}+T_{b}^{-1}\right)^{2}},
$$

we can found that the value of $n$ that maximizes PoS is $n=2$; in this case

$$
\operatorname{PoS}=\frac{4 T_{b}^{-1}-3 T_{b}^{1}+T_{a}^{1}}{3 T_{b}^{-1}-2 T_{b}^{1}+T_{a}^{1}} .
$$

Independently of the values of $T_{a}^{1}, T_{b}^{1}$, and $T_{b}^{-1}$ the upper bound for $\mathrm{PoS}$ is $\frac{4}{3}=1 . \overline{3}$. We consider the settings where $n=\infty$. Since $\Psi$ and $F$ are very similar, we can write $\Psi<a \cdot F$ where $a=\min _{i}\left\{\frac{x^{i}+1}{x^{i}}\right\}$. This expression can be used to provide an upper bound on PoA. Precisely, PoS < $\min _{i}\left\{\frac{x^{i}+1}{x^{i}}\right\}$. Easily, as $x^{i}$ goes to $\infty$, PoS goes to 1 .

We study the bounds on PoA. When $n<\infty$, the proof is similar to the one discussed in the previous section. We report just the sketch of the proof. The worst topology class is the "corridor" and the worst "corridor" topology holds to be the one with $m=3$ and $n=3$. It can be observed that PoA is maximum when either $\lambda_{2}=0$ or all the rates are infinity (and their reverse is zero). Obviously, the maximum PoA holds to be $1 . \overline{6}$. When $n=\infty$, the values of PoA and PoS are the same and then PoA is 1 .

\subsection{Multiplicative Interference-Rate Cost Func- tion}

We consider the case in which the cost functions are given by the multiplication between the number of interferers and the reverse of the rate perceived by users. Formally, users' cost functions are: $c_{j}\left(i, x^{i}\right)=T_{j}^{i} \cdot x^{i}$. The game does not admit exact potential function.

We analyze PoA and PoS in this specific setting, with objective function $F$ defined as social cost. We state the following proposition.

Proposition 4.3. The upper bounds for PoA and PoS are: $\operatorname{PoA}(n<\infty) \leq \frac{\max _{i, j} T_{j}^{i}+\min _{i, j} T_{j}^{i}}{\min _{i, j} T_{j}^{i}}, \operatorname{PoA}(n=\infty) \leq$ $\frac{\max _{i, j} T_{j}^{i}+\min _{i, j} T_{j}^{i}}{\min _{i, j} T_{j}^{i}}, \operatorname{PoS}(n<\infty)<\frac{\max _{i, j} T_{j}^{i}+\min _{i, j} T_{j}^{i}}{\min _{i, j} T_{j}^{i}}$, and $\operatorname{PoS}(n=\infty) \leq \frac{\max _{i, j} T_{j}^{i}+\min _{i, j} T_{j}^{i}}{\min _{i, j} T_{j}^{i}}$.

Proof. We start by proving the bound on PoS when $n<\infty$. We consider the same setting we considered in the previous section to prove the bound on PoS. Here we assume that $n T_{b}^{1}<T_{b}^{-1}$. The unique equilibrium prescribes $x^{1}=n$ and $x^{i}=0$ for all $i \in\{2, \ldots, m\}$. The optimal allocation that maximizes PoS prescribes $x^{i}=1$ for all $i$. We can write PoS as:

$$
\mathrm{PoS}=\frac{n T_{a}^{1}+n \cdot(n-1) T_{b}^{1}}{T_{a}^{1}+(n-1) T_{b}^{-1}}
$$

Since $n T_{b}^{1}<T_{b}^{-1}$, we can write

$$
\operatorname{PoS}<\frac{n T_{a}^{1}+(n-1) T_{b}^{-1}}{T_{a}^{1}+(n-1) T_{b}^{-1}} .
$$

By studying the derivative of the bound with respect to $n$, i.e.

$$
\frac{d \mathrm{PoS}}{d n}=\frac{T_{a}^{1} \cdot T_{a}^{1}}{\left(T_{a}^{1}+(n-1) T_{b}^{-1}\right)^{2}},
$$

it can be observed that the bound is strictly monotonically increasing in $n$. The upper bound is when $n \rightarrow \infty$ and it is $\frac{T_{a}^{1}+T_{b}^{-1}}{T_{b}^{-1}}$. In a general setting, an upper bound for PoS can be computed by replacing $T_{a}^{1}$ with $\max _{i, j} T_{j}^{i}$ and $T_{b}^{-1}$ with $\min _{i, j} T_{j}^{i}$. Notice that the bound on PoS is equal to or greater than 2 .

We study PoA. When $n<\infty$, by the analysis of "corridor" topologies with $m>2$, it can be observed that no topology admits a bound on PoA greater than the bound with $m=2$. The upper bound on PoA is exactly the upper bound on PoS, i.e.

$$
\mathrm{PoA} \leq \frac{\max _{i, j} T_{j}^{i}+\min _{i, j} T_{j}^{i}}{\min _{i, j} T_{j}^{i}} .
$$

Notice that, while PoS is strictly lower than the above upper bound, PoA can reach such a bound. When $n=\infty$, the values of PoA and PoS are the same.

\section{NUMERICAL RESULTS}

In this section, we complement the theoretical analysis previously discussed by providing a mathematical programming formulation to compute Nash equilibria in realistic settings. We use the proposed model to compare the quality of the equilibria induced by the three different cost functions we introduced in Section 4.

\subsection{Mathematical Programming Formulation}

We derive hereafter a mathematical programming formulation to find Nash equilibria. We introduce the following parameter:

$$
a_{j i}=\left\{\begin{array}{ll}
1 & \text { if user } j \text { can choose } A_{i} \\
0 & \text { otherwise }
\end{array} .\right.
$$

Given a generic topology, $a_{j i}$ is equal to 1 if user $j$ is within the coverage area of $A_{i}$. Coverage is defined in the following way: let $P_{t x}^{i}$ be the power transmitted by $A_{i}$; user $j$ is covered by $A_{i}$ if the following inequality holds:

$$
P_{j}^{i}=\alpha P_{t x}^{i} d_{i j}^{\eta} 10^{\frac{\epsilon}{10}} \geq P_{t h},
$$

where $d_{i j}$ is the distance between $A_{i}$ and user $j, \eta$ is the attenuation factor, and $10^{\frac{\epsilon}{10}}$ accounts for the loss due to slow shadowing, being $\epsilon$ a normal variate with zero mean and $\sigma^{2}$ variance. $P_{t h}$ is a threshold value for the required received power. ${ }^{3}$

We define the assignment of a user to an access point by introducing a binary decision variable:

$$
y_{j i}= \begin{cases}1 & \text { if user } j \text { chooses } A_{i} \\ 0 & \text { otherwise }\end{cases}
$$

Finally, the constraints of the problem are:

$$
\sum_{i \in \mathcal{A}} y_{j i}=1 \quad \forall j \in \mathcal{N}
$$

$$
y_{j i} \leq a_{j i} \quad \forall j \in \mathcal{N}, i \in \mathcal{A},
$$

\footnotetext{
${ }^{3}$ It is worth noting here that the mathematical programming formulation is general and absolutely independent on the specific propagation/coverage model
} 


$$
a_{j k} y_{j i} c_{j}\left(i, \sum_{l \in \mathcal{N}} y_{l i}\right) \leq c_{j}\left(k, \sum_{l \in \mathcal{N}} y_{l k}\right), \quad \forall j \in \mathcal{N}, i, k \in \mathcal{A}
$$

Constraints (1) ensure that each user chooses only one access network. Constraints (2) guarantee the feasibility of the assignment. Constraints (3) force each user to choose the strategy (access network) which leads to the minimum cost function, that is, they ensure that, if the single user unilaterally changes her strategy, the change does not improve her own payoff (i.e. definition of Nash equilibrium).

Notice that the expression of the cost function in (3) is the same defined previously:

$$
x^{i}=\sum_{l \in \mathcal{N}} y_{l i}
$$

This formulation allows one to find equilibria that maximize/minimize a given objective function. In our analysis we are interested in finding both equilibria that minimize and equilibria that maximize the social cost. They can be found by introducing the following objective function in the formulation:

$$
\min / \max \sum_{j \in \mathcal{N}} \sum_{i \in \mathcal{A}} y_{j i} c_{j}\left(i, \sum_{l \in \mathcal{N}} y_{l i}\right) .
$$

Moreover, to evaluate price-of-stability and price-of-anarchy, it is necessary to provide a model that allows one to find the optimal solution, i.e. the solution that minimizes the social cost but that could not be an equilibrium. To do this we inhibit equilibrium constraints (3) and solve the model minimizing the objective function (4).

Constraints (3) and objective function (4) must be instantiated according to the specific adopted cost function. For the sake of brevity, we report in Appendix B the formulas for the additive interference-rate cost function. ${ }^{4}$

\subsection{Experimental Evaluation}

In this subsection we report some numerical results in order to compare the strategies of users when they use the three different cost functions. We have implemented an instance generator able to create synthetic instances representing multi-access network scenarios. The software takes as input the following parameters: the edge of the square area to be simulated $(L)$, the number of end users $(n)$, the weight $(\lambda)$ used in the second cost function (supposing that $\lambda=\lambda_{2}$ and $\left.1-\lambda=\lambda_{1}\right)$, the number of access points $(m)$, and the coverage range of each access point, expressed in meters $(r)$. In a basic set of instances, each network is assumed to have a circular coverage area with radius $r=100$ meters.

The generating tool randomly draws the position of the $m$ access points and of the $n$ users, so that each user is covered by at least one access point. According to the distance between the user and the access point, when this is shorter than the radius, the software assigns the larger achievable rate that the user can obtain from that access point. In our scenarios we suppose to have a $802.11 \mathrm{~g}$ network. Table 1 reports, for each distance, the achievable rate and the corresponding value of $T$ used in our simulations. The parameter $T$ used in the model is a normalization of the inverse of rate $R$, so that the number of interferers and the inverse of rate

${ }^{4}$ The instantiation of the proposed model with the cost functions presented in Sections 4.1 and 4.2 is a integer linear problem (ILP), while with the cost function presented in Section 4.3 the model is a mixed integer linear problem (MILP).
Table 1: Achievable rates in a $802.11 \mathrm{~g}$ scenario.

\begin{tabular}{|c|c|c|}
\hline $\begin{array}{c}\text { Distance } \\
{[\text { meters }]}\end{array}$ & $\begin{array}{c}\text { Rate } \\
{[\text { Mbit } / \mathrm{s}]}\end{array}$ & $\mathrm{T}$ \\
\hline$\leq 10$ & 54 & 1.8 \\
\hline$\leq 20$ & 48 & 2 \\
\hline$\leq 30$ & 36 & 2.7 \\
\hline$\leq 45$ & 24 & 4 \\
\hline$\leq 60$ & 18 & 5.5 \\
\hline$\leq 75$ & 12 & 8.3 \\
\hline$\leq 90$ & 9 & 11.1 \\
\hline$\leq 100$ & 6 & 16.6 \\
\hline
\end{tabular}

are comparable. In this case we assume that $T=10^{8} / R$. This normalization could not be effective if the number of users or access points increases. In general, we have found that an effective normalization should depend on $n$ and $m$ so that the worst case of rate (that corresponds to the maximum value of $T$ ) is comparable with the average number of users per access point. For this reason we propose the following normalization:

$$
T_{\max }=\frac{n}{m} \beta
$$

where $T_{\max }$ is the maximum value of $T$ and $\beta$ should be chosen in the interval $1 \div 4$ (in our simulations $\beta=3.32$ ).

All the results reported in the remainder have been produced formalizing the mathematical problem presented in Section 5.1 in AMPL [9] and solving it with CPLEX commercial solver [1]. Unless differently specified, the reported results are averaged on 100 randomly generated instances.

Table 2 reports the results obtained in a uniform topology with $m=10$ randomly deployed access points and $n=50$ end users, in case $L=500$ meters and $r=100$ meters. For each scenario we have considered the three different cost functions. With $1^{s t}$ we indicate the cost function presented in Section 4.1, while $2^{\text {nd }}$ is the cost function presented in Section 4.2 and $3^{\text {rd }}$ the one presented in Section 4.3 .

The average number of interferers and the average rate (in Mbit/s) per user are reported for the best equilibrium, the worst equilibrium, and the optimal solution. The last two columns report the value of price-of-stability and priceof-anarchy.

The result coming from this analysis is the different behavior of users, minimizing different cost functions. When users aim at minimizing the number of interferers $\left(1^{\text {st }}\right.$ function) they obtain the optimal solution from the point of view of interference, ignoring the achievable rates. This solution is the same that can be obtained setting $\lambda=0$ in the $2^{\text {nd }}$ cost function. By increasing $\lambda$, users give more importance to rate with respect to interference. When $\lambda=1.0$ the problem is not a game anymore, because each user simply chooses the access point that guarantees the highest rate (for this reason $\mathrm{PoS}=\mathrm{PoA}=1)$. Using the $3^{\text {rd }}$ cost function, users obtain a trade off between interference and rate.

We have also evaluated the price-of-anarchy in a simple scenario, showing that it decreases quickly as the number of users increases. For the reasons mentioned in Section 4.1.2, we consider a "corridor" topology composed by $m=3$ access points with $r=100$ meters, varying the number of users from 3 to 50. For each cost function, we report average and maximum value of price-of-anarchy.

Fig. 6 reports the price-of-anarchy using the $1^{\text {st }}$ cost function. Results confirm what we have formally proved in Section 4.1. Namely, the maximum value of PoA is $1 . \overline{6}$ and 
Table 2: PoA and PoS with different cost functions in a uniform network scenario with $n=50$ users, $m=10$ access points, $L=500 \mathrm{~m}$, and $r=100 \mathrm{~m}$.

\begin{tabular}{|c|c|c|c|c|c|c|c|c|c|}
\hline & & \multicolumn{2}{|c|}{ Best equilibrium } & \multicolumn{2}{c|}{ Worst equilibrium } & Optimal solution & PoS & PoA \\
\hline & & Interf. & Rate & Interf. & Rate & Interf. & Rate & & \\
\hline $1^{\text {st }}$ & & 5.652 & 16.542 & 5.708 & 16.614 & 5.652 & 16.158 & 1.00000 & 1.01049 \\
\hline \hline $2^{\text {nd }}$ & $\lambda=0.1$ & 5.664 & 18.306 & 5.700 & 17.016 & 5.656 & 18.234 & 1.00039 & 1.01628 \\
\hline & $\lambda=0.2$ & 5.696 & 18.558 & 5.756 & 18.402 & 5.672 & 18.432 & 1.00094 & 1.01067 \\
\hline & $\lambda=0.3$ & 5.768 & 18.948 & 5.812 & 18.774 & 5.692 & 18.552 & 1.00198 & 1.00760 \\
\hline & $\lambda=0.4$ & 5.844 & 19.188 & 5.892 & 19.062 & 5.756 & 18.960 & 1.00222 & 1.00630 \\
\hline & $\lambda=0.5$ & 5.960 & 19.470 & 5.988 & 19.434 & 5.804 & 19.074 & 1.00288 & 1.00501 \\
\hline & $\lambda=0.6$ & 6.064 & 19.584 & 6.076 & 19.596 & 5.936 & 19.356 & 1.00228 & 1.00267 \\
\hline & $\lambda=0.7$ & 6.100 & 19.632 & 6.100 & 19.632 & 6.000 & 19.506 & 1.00089 & 1.00089 \\
\hline & $\lambda=0.8$ & 6.116 & 19.644 & 6.116 & 19.644 & 6.076 & 19.596 & 1.00016 & 1.00016 \\
\hline & $\lambda=0.9$ & 6.144 & 19.656 & 6.144 & 19.656 & 6.116 & 19.644 & 1.00001 & 1.00001 \\
\hline & $\lambda=1.0$ & 6.348 & 19.656 & 6.348 & 19.656 & 6.348 & 19.656 & 1.00000 & 1.00000 \\
\hline \hline $3^{\text {rd }}$ & & 5.856 & 19.344 & 5.888 & 19.320 & 5.764 & 18.888 & 1.01214 & 1.02180 \\
\hline
\end{tabular}

it can be reached only with 3 users, moreover PoA is different from 1 only when the number of users is a multiple of the number of access points. We have also reported the analytical bound:

$$
P o A=\frac{n^{2}+6}{n^{2}}
$$

derived in Appendix A.

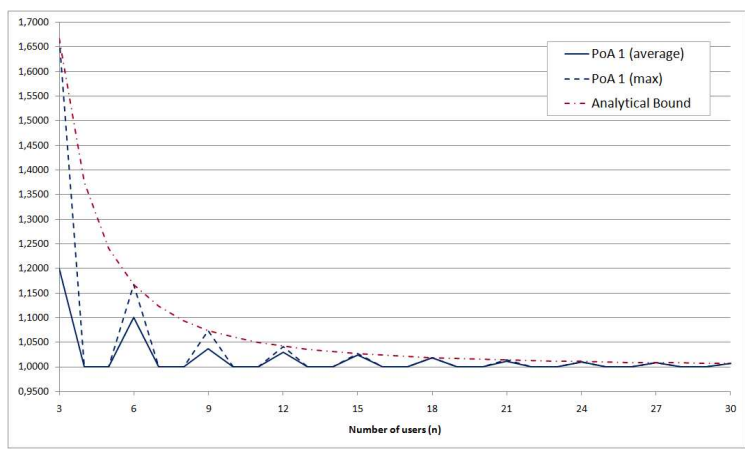

Figure 6: PoA using the $1^{\text {st }}$ cost function.

Fig. 7 and 8 report PoA using the $2^{\text {nd }}$ and $3^{\text {rd }}$ cost function. Also in these cases, we can observe that it rapidly decreases and becomes very close to one. The same experimental results can be produced considering PoS.

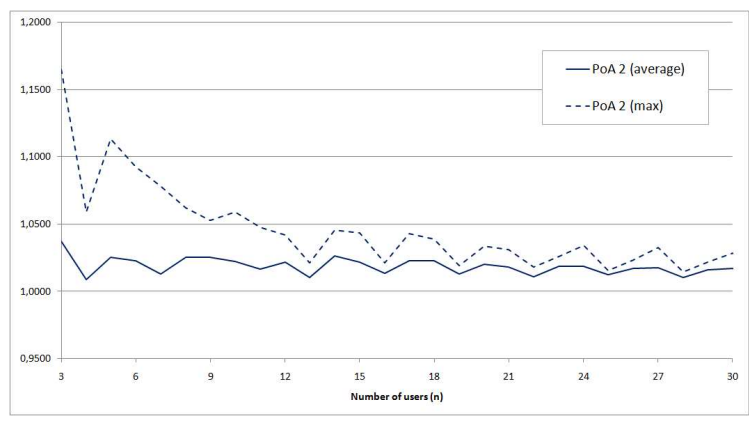

Figure 7: PoA using the $2^{\text {nd }}$ cost function.

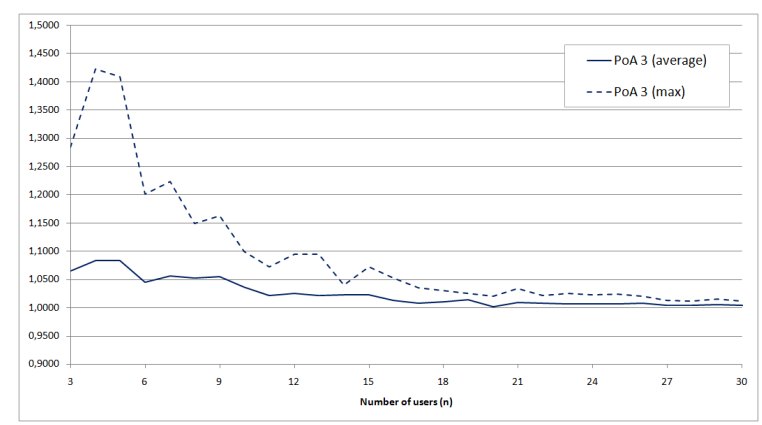

Figure 8: PoA using the $3^{r d}$ cost function.

Summarily, although the theoretical bounds on PoA and PoS derived in Section 4 are generally far from 1 , in realistic scenarios where access points and users are uniformly distributed, the values of PoA and PoS are very close to 1 independently of the adopted cost function. Further analysis must be directed to consider non-uniformly topologies and to compare the equilibria induced by the cost functions in terms of actual throughput through system level simulation.

\section{CONCLUDING REMARKS AND FUTURE WORK}

The problem of network selection naturally arises in those wireless access network scenarios where mobile users are offered multiple connectivity opportunities provided by different access networks/technologies. In this scenario, we have analyzed the dynamics of a competitive network selection scenario, where mobile users select the accessing network selfishly minimizing their own selection cost. We have casted the problem as a non-cooperative game belonging to the class of congestion games, and we have analyzed the quality of the corresponding Nash equilibria under three different expressions for the selection cost depending on the interference level and the nominal throughput.

The analysis has been carried out in two ways: first, we have derived analytical upper bounds for the price-ofanarchy and price-of-stability, which are considerably tighter than well known bounds for generic congestion games; second, we have developed a mathematical programming model 
to numerically calculate the Nash equilibria, providing an operational tool to validate the analytical results and "solve" the game for realistic wireless access network topologies.

We are currently working in two directions: evaluating different expressions for the selection cost and characterizing the game equilibria through system level simulation, which allows one to gather networking-oriented performance measures (e.g., actual throughput and delivery delay).

\section{REFERENCES}

[1] ILOG CPLEX 10.0 user's manual. http://www.ilog.com/products/cplex/.

[2] E. Anshelevich, A. Dasgupta, J. Kleinberg, E. Tardos, T.Wexler, and T. Roughgarden. The price of stability for network design with fair cost allocation. In Proc. IEEE FOCS, pages 59-73, Rome, Italy, October 17-19 2004.

[3] B. Awerbuch, Y. Azar, and L. Epstein. The price of routing unsplittable flow. In Proceedings of Symposium on Theory of Computing (STOC), pages 57-66, Baltimore, USA, May 21-24 2005.

[4] M. J. Beckmann, C. B. McGuire, and C. B. Winstein. Studies in the Economic Transportation. Yale University Press, 1956.

[5] M. Bernaschi, F. Cacace, G. Iannello, S. Za, and A. Pescape. Seamless internetworking of wlans and cellular networks: architecture and performance issues in a mobile ipv6 scenario. IEEE Wireless Communications, 12(3):73-80, June 2005.

[6] N. Blefari-Melazzia, D. D. Sorte, M. Femminella, and G. Reali. Autonomic control and personalization of a wireless access network. Computer Networks, 51(10):2645-2676, July 2007.

[7] M. Cesana, I. Malanchini, and A. Capone. Modelling network selection and resource allocation in wireless access networks with non-cooperative games. to appear in IEEE MASS 2008.

[8] D. Charilasa, O. Markakia, D. Nikitopoulos, and M. Theologoua. Packet-switched network selection with the highest qos in $4 \mathrm{~g}$ networks. Computer Networks, 52(1):248-258, 2008.

[9] R. Fourer, D. M. Gay, and B. W. Kernighan. AMPL, A modeling language for mathematical programming. 1993.

[10] D. Fudenberg and J. Tirole. Game Theory. The MIT Press, Cambridge, USA, 1991.

[11] E. Gustafsson and A. Jonsson. Always best connected. IEEE Wireless Communications, 10:49-55, 2003.

[12] E. Koutsoupias and C. H. Papadimitriou. Worst-case equilibria. In Proc. STACS, pages 404-413, Trier, Germany, March 4-6 1999.

[13] Y. Lee and S. C. Miller. Network selection and discovery of service information in public wlan hotspots. In Proc. ACM WMASH, pages 81-92, Philadelphia, USA, October 12004.

[14] I. Milchtaich. Congestion games with player-specfic payoff functions. Games and Economic Behavior, 13(1):111-124, 1996.

[15] O. Ormond, J. Murphy, and G. Muntean. Utility-based intelligent network selection in beyond 3g systems. In Proc. IEEE ICC, pages 1831-1836, Instanbul, Turkey, June 11-15 2006.
[16] R. W. Rosenthal. A class of games possessing pure-strategy Nash equilibria. International Journal of Game Theory, 2(1):65-67, 1973.

[17] Q. Song and A. Jamalipour. Network selection in an integrated wireless lan and umts environment using mathematical modelling and computing techniques. IEEE Wireless Communications, 12(3):42-48, June 2005.

[18] J. G. Wardrop. Some theoretical aspects of road traffic research. Proceedings of Institute of Civil Engineers, 1(2):325-378, 1952.

\section{APPENDIX}

\section{A. EXAMPLE OF APPLICATION OF AL- GORITHM 1}

We solve the inequality system:

$$
\left\{\begin{array}{l}
n_{1}+x_{1,2}^{1} \leq n_{2}+x_{1,2}^{2}+x_{2,3}^{2}+1 \\
n_{2}+x_{1,2}^{2}+x_{2,3}^{2} \leq n_{1}+x_{1,2}^{1}+1 \\
n_{2}+x_{1,2}^{2}+x_{2,3}^{2} \leq n_{3}+x_{2,3}^{3}+1 \\
n_{3}+x_{2,3}^{3} \leq n_{2}+x_{1,2}^{2}+x_{2,3}^{2}+1
\end{array} .\right.
$$

Letting $A=n_{1}+x_{1_{2}}^{1}, B=n_{2}+x_{1,2}^{1}+x_{2,3}^{2}, C=n_{3}+x_{2,3}^{2}$, we obtain:

$$
\left\{\begin{array}{l}
B-1 \leq A \leq B+1 \\
C-1 \leq B \leq C+1
\end{array}\right.
$$

Four cases are possible.

1. $(A=B=C)$ Since $n=A+B+C$, we have $A=B=$ $C=n / 3$. The solution is:

$$
\left\{\begin{array}{l}
x_{1,2}^{1}=\frac{n}{3}-n_{1} \\
x_{1,2}^{2}+x_{2,3}^{2}=\frac{n}{3}-n_{2} \\
x_{2,3}^{3}=\frac{n}{3}-n_{3}
\end{array} .\right.
$$

2. $(A=B=C+1$ or $B=C=A+1$ or $A=C=B+1)$ In this case $n=3 A-1=3 B-1=3 C+2$. The solution is of the form:

$$
\left\{\begin{array}{l}
x_{1,2}^{1}=\frac{n+1}{3}-n_{1} \\
x_{1,2}^{2}+x_{2,3}^{2}=\frac{n+1}{3}-n_{2} \\
x_{2,3}^{3}=\frac{n-2}{3}-n_{3}
\end{array} .\right.
$$

3. $(A=B=C-1$ or $A=C=B-1$ or $B=C=A-1)$ In this case $n=3 A-1=3 B-1=3 C+2$. The solution is of the form:

$$
\left\{\begin{array}{l}
x_{1,2}^{1}=\frac{n-1}{3}-n_{1} \\
x_{1,2}^{2}+x_{2,3}^{2}=\frac{n-1}{3}-n_{2} \\
x_{2,3}^{3}=\frac{n+2}{3}-n_{3}
\end{array} .\right.
$$

4. $(A=B-1=C-2$ or $A=B+1=C+2)$ In this case $n=3 A+3=3 B=3 C-3$. The solution is of the form:

$$
\left\{\begin{array}{l}
x_{1,2}^{1}=\frac{n-3}{3}-n_{1} \\
x_{1,2}^{2}+x_{2,3}^{2}=\frac{n}{3}-n_{2} \\
x_{2,3}^{3}=\frac{n+3}{3}-n_{3}
\end{array} .\right.
$$

If all variables are positive, a solution has been found. In the case $n$ is divisible by $m$, there are two equilibria with different social costs. In this case, the price-of-anarchy is:

$$
\mathrm{PoA}=\frac{\left(\frac{n-3}{3}\right)^{2}+\left(\frac{n}{3}\right)^{2}+\left(\frac{n+3}{3}\right)^{2}}{3\left(\frac{n}{3}\right)^{2}}=\frac{n^{2}+6}{n^{2}} .
$$

Instead, if at least one variable is negative, we set it equal to zero and we solve the previous system after having removed the pertinent inequalities. 
For instance, if $x_{2,3}^{3} \leq 0$, we set it equal to zero and we remove the possibility of choosing $A_{3}$ for all the users in $N_{2,3}$. That is, all the users in $N_{2,3}$ will choose $A_{2}$, formally, $x_{2,3}^{2}=n_{2,3}$. In this case the new problem to solve is simpler than the previous one, since both the number of variables and the number of inequality decrease by two. We call $n^{\prime}=$ $n_{1}+n_{1,2}+n_{2}+n_{2,3}$. The non-deviation inequality system is:

$$
\left\{\begin{array}{l}
n_{1}+x_{1,2}^{1} \leq n_{2}+x_{1,2}^{2}+n_{2,3}+1 \\
n_{2}+x_{1,2}^{2}+n_{2,3} \leq n_{1}+x_{1,2}^{1}+1
\end{array} .\right.
$$

Letting $A=n_{1}+x_{1,2}^{1}$ and $B=n_{2}+x_{1,2}^{2}+n_{2,3}$, we obtain:

$$
B-1 \leq A \leq B+1
$$

Two cases are possible.

1. $(A=B)$ In this case $A=B=n^{\prime} / 2$. The solution is:

$$
\left\{\begin{array}{l}
x_{1,2}^{1}=\frac{n^{\prime}}{2}-n_{1} \\
x_{1,2}^{2}=\frac{n^{\prime}}{2}-n_{2}-n_{2,3}
\end{array}\right.
$$

2. $(A=B+1$ or $B=A+1)$ In this case $n^{\prime}=2 A-1=$ $2 B+1$. The solution is of the form:

$$
\left\{\begin{array}{l}
x_{1,2}^{1}=\frac{n^{\prime}+1}{{ }^{2}}-n_{1} \\
x_{1,2}^{2}=\frac{n^{\prime}-1}{2}-n_{2}-n_{2,3}
\end{array}\right.
$$

If either $x_{1,2}^{1}$ or $x_{1,2}^{2}$ is non-positive, we set this variable equal to zero and we produce the pertinent inequality system.

\section{B. MATHEMATICAL FORMULATION}

We present here the mathematical formulation used for the second cost function. We omit variables and constraints that are the same presented in Section 5.1.

We report the equilibrium constraint (3) and the objective function (4), previously presented in a general form.

$$
\begin{aligned}
& M\left(y_{j i} a_{j k}-1\right)+\left(\lambda_{1} \sum_{l \in \mathcal{N}, l \neq j} y_{l i}+\lambda_{2} T_{j}^{i}\right) \leq \\
& \left(\lambda_{1} \sum_{l \in \mathcal{N}, l \neq j} y_{l k}+\lambda_{2} T_{j}^{k}\right) \quad \forall j \in \mathcal{N}, i, k \in \mathcal{A}
\end{aligned}
$$

This "big-M" constraint is the linearized form of (3) and is activated only when $y_{j i}=1$ and $a_{j k}=1$. In other words, player $j$ chooses $A_{i}, y_{j i}=1$, if all the other possible strategies, access points $A_{k}$ for which $a_{j k}=1$, give an higher cost to $j$.

The objective function (4) can be written as:

$$
\min / \max \sum_{j \in \mathcal{N}}\left(\lambda_{1} \sum_{i \in \mathcal{A}} y_{j i} \sum_{l \in \mathcal{N}} y_{l i}+\lambda_{2} \sum_{i \in \mathcal{A}} T_{j}^{i} y_{j i}\right)
$$

To linearize the objective function we have to introduce a new variable $\left(v_{j} \in \mathbb{N}, \forall j \in \mathcal{N}\right)$. In the case of minimization we have:

$$
\begin{gathered}
\min _{j \in \mathcal{N}}\left(\lambda_{1} v_{j}+\lambda_{2} \sum_{i \in \mathcal{A}} T_{j}^{i} y_{j i}\right) \\
v_{j} \geq \sum_{l \in \mathcal{N}} y_{l i}+M\left(y_{j i}-1\right) \quad \forall j \in \mathcal{N}, i \in \mathcal{A}
\end{gathered}
$$

The linearization of the objective function in the case of maximization is analogous. 